Proc Indian Natn Sci Acad 81 No. 1 February 2015 Special Issue, pp. 62-69

(C) Printed in India.

DOI: $10.16943 /$ ptinsa/2015/v81i1/48052

\title{
Relativistic Third-order Viscous Hydrodynamics
}

\author{
AMARESH JAISWAL ${ }^{*}$ \\ Tata Institute of Fundamental Research, Mumbai 400 005, India
}

(Received on 25 April 2014; Accepted on 18 June 2014)

\begin{abstract}
Employing the iterative solution of Boltzmann equation in relaxation time approximation, we derive a third-order evolution equation for shear stress tensor from its kinetic definition. To this end we first obtain the non-equilibrium phase-space distribution function, $f(x, p)$, up to second-order in gradients. The expression for $\delta f(x, p)$ thus obtained does not lead to the violation of the experimentally observed $1 / \sqrt{m_{T}}$ scaling of the longitudinal femtoscopic radii, as is the case with the widely used Grad's approximation, and hence is better suited for hydrodynamic modelling of relativistic heavy-ion collisions. Subsequently, we quantify the significance of this new derivation within one-dimensional scaling expansion and demonstrate that the results obtained using third-order viscous equations are in excellent agreement with the exact solution of Boltzmann equation as well as transport results.
\end{abstract}

\section{Key Words : Relativistic Hydrodynamics; Kinetic Theory; Boltzmann Equation; Shear Stress Tensor}

\section{Introduction}

The long-wavelength, low frequency limit of the microscopic dynamics leads to an effective hydrodynamical description of the system. The collective behaviour of the hot and dense, strongly interacting matter, created in high-energy heavy-ion collisions, has been studied quite extensively within the framework of relativistic hydrodynamics. The theory of relativistic hydrodynamics is formulated as a gradient expansion where ideal hydrodynamics is zeroth-order. However, as all fluids are inherently dissipative by virtue of uncertainty principle (Danielewicz and Gyulassy, 1985), the dissipative effects can not be ignored. Although the firstorder theories, collectively known as relativistic Navier-Stokes theory (Eckart, 1940; Landau and Lifshitz, 1987) incorporate dissipation, however they suffer from acausality and numerical instability. The secondorder, Israel-Stewart (IS) theory (Israel and Stewart, 1979) solves the acausality problem (Huovinen and Molnar, 2009) but may not guarantee stability.

\footnotetext{
*Author for Correspondence : E-mail: amaresh.tifr@gmail.com
} 
The IS theory has been applied quite successfully in explaining a wide range of collective phenomena observed in high-energy heavy-ion collision experiments at Relativistic Heavy-Ion Collider (RHIC) and recently at Large Hadron Collider (LHC). Despite its success, the formulation of IS theory is based on strong assumptions and approximations, namely: use of second moment of Boltzmann equation and the Grad's 14-moment approximation (Israel and Stewart, 1979). While it was shown later that the dissipative equations can be obtained directly from their definitions without resorting to the second-moment of Boltzmann equation (Denicol et al., 2010), it has been shown recently that both these assumptions are unnecessary and instead of 14-moment approximation, iterative solution of Boltzmann equation can be employed to derive the dissipative evolution equations (Jaiswal, 2013a).

Apart from these theoretical issues in the formulation, IS theory suffers from several other shortcomings on the phenomenological level. In the case of one-dimensional scaling expansion (Bjorken, 1983), IS theory has resulted in unphysical effects such as reheating of the expanding medium (Muronga, 2004) and negative longitudinal pressure (Martinez and Strickland, 2009). Moreover, comparison of IS equations with transport results show disagreement for $\eta / s>0.5$ indicating the breakdown of second-order theory (Huovinen and Molnar, 2009; El et al., 2009). Furthermore, inclusion of dissipative corrections to the phase-spcae distribution function, $f(x, p)$, via Grad's 14-moment approximation leads to the violation of experimentally observed $1 / \sqrt{m_{T}}$ scaling of the longitudinal Hanbury Brown-Twiss (HBT) radii.

To extend the range of applicability of the IS equations, second-order dissipative equations were derived from Boltzmann equation where the collision term was generalized to include nonlocal effects through gradients of $f(x, p)$ (Jaiswal, et al., 2013a). Moreover, it was shown that the inclusion of third-order corrections to the evolution equation of shear stress tensor led to an improved agreement with transport results (El et al., 2010; Jaiswal, 2013b). Furthermore, a general moment method was devised to improve Grad's 14-moment approximation beyond its current scope by introducing orthogonal basis in momentum expansion (Denicol et al., 2012). The correct and consistent formulation of relativistic viscous hydrodynamics is not yet conclusively settled and is currently a topic of intense investigation (Denicol et al., 2010; Jaiswal 2013a; Jaiswal et al., 2013b; El et al., 2010; Jaiswal, 2014; Jaiswal et al., 2014; Denicol et al., 2012; Jaiswal et al., 2013c; Bhalerao et al., 2013, 2014).

\section{Relativistic Hydrodynamics}

The equation of motion governing the hydrodynamic evolution of a relativistic system with no net conserved charges is obtained from the local conservation of energy and momentum, $\partial_{\mu} T^{\mu \nu}=0$. In terms of singleparticle phase-space distribution function, the energy-momentum tensor of a macroscopic system can be 
expressed as (deGroot et al., 1980)

$$
T^{\mu \nu}=\int d p p^{\mu} p^{\nu} f(x, p)=\epsilon u^{\mu} u^{\nu}-P \Delta^{\mu \nu}+\pi^{\mu \nu}
$$

where $d p \equiv g d \mathbf{p} /\left[(2 \pi)^{3}|\mathbf{p}|\right], g$ being the degeneracy factor, $p^{\mu}$ is the particle four-momentum, and $f(x, p)$ is the phase-space distribution function. In the tensor decomposition, $\epsilon, P$, and $\pi^{\mu \nu}$ are energy density, thermodynamic pressure, and shear stress tensor, respectively. The projection operator $\Delta^{\mu \nu} \equiv g^{\mu \nu}-u^{\mu} u^{\nu}$ is orthogonal to the hydrodynamic four-velocity $u^{\mu}$ defined in the Landau frame: $T^{\mu \nu} u_{\nu}=\epsilon u^{\mu}$. The metric tensor is Minkowskian, $g^{\mu \nu} \equiv \operatorname{diag}(+,-,-,-)$. Here we have restricted ourselves to a system of massless particles (ultrarelativistic limit) for which the bulk viscosity vanishes.

The conservation of the energy-momentum tensor, when projected along and orthogonal to $u^{\mu}$, leads to the evolution equations for $\epsilon$ and $u^{\mu}$ :

$$
\dot{\epsilon}+(\epsilon+P) \theta-\pi^{\mu \nu} \nabla_{(\mu} u_{\nu)}=0, \quad(\epsilon+P) \dot{u}^{\alpha}-\nabla^{\alpha} P+\Delta_{\nu}^{\alpha} \partial_{\mu} \pi^{\mu \nu}=0
$$

where we employ the standard notation $\dot{A} \equiv u^{\mu} \partial_{\mu} A$ for comoving derivative, $\theta \equiv \partial_{\mu} u^{\mu}$ for expansion scalar, $A^{(\alpha} B^{\beta)} \equiv\left(A^{\alpha} B^{\beta}+A^{\beta} B^{\alpha}\right) / 2$ for symmetrization, and $\nabla^{\alpha} \equiv \Delta^{\mu \alpha} \partial_{\mu}$ for space-like derivatives. For the massless case, the equation of state relating energy density and pressure is $\epsilon=3 P \propto \beta^{-4}$. The Landau matching condition $\epsilon=\epsilon_{0}$ is employed to fix the inverse temperature, $\beta \equiv 1 / T$, where $\epsilon_{0}$ is the equilibrium energy density. The derivatives of $\beta$,

$$
\dot{\beta}=\frac{\beta}{3} \theta-\frac{\beta}{12 P} \pi^{\rho \gamma} \sigma_{\rho \gamma}, \quad \nabla^{\alpha} \beta=-\beta \dot{u}^{\alpha}-\frac{\beta}{4 P} \Delta_{\rho}^{\alpha} \partial_{\gamma} \pi^{\rho \gamma}
$$

can be obtained from Eq. (2), where $\sigma^{\rho \gamma} \equiv \nabla^{(\rho} u^{\gamma)}-(\theta / 3) \Delta^{\rho \gamma}$ is the velocity stress tensor (Jaiswal, 2013b).

When the system is close to local thermodynamic equilibrium, the distribution function can be written as $f=f_{0}+\delta f$, where $\delta f \ll f_{0}, f_{0}=\exp (-\beta u \cdot p)$ is the equilibrium distribution function of Boltzmann particles at vanishing chemical potential and $u . p \equiv u_{\mu} p^{\mu}$. Projecting the traceless symmetric part of Eq. (1), we obtain an expression for the shear stress tensor and its time evolution,

$$
\pi^{\mu \nu}=\Delta_{\alpha \beta}^{\mu \nu} \int d p p^{\alpha} p^{\beta} \delta f, \quad \dot{\pi}^{\langle\mu \nu\rangle}=\Delta_{\alpha \beta}^{\mu \nu} \int d p p^{\alpha} p^{\beta} \delta \dot{f}
$$

where $\Delta_{\alpha \beta}^{\mu \nu} \equiv \Delta_{(\alpha}^{\mu} \Delta_{\beta)}^{\nu}-(1 / 3) \Delta^{\mu \nu} \Delta_{\alpha \beta}$. In the following, we iteratively solve the Boltzmann equation to determine $\delta f$ and subsequently derive evolution equation for shear stress tensor. 


\section{Viscous Evolution Equations}

We start from the relativistic Boltzmann equation with the relaxation-time approximation for the collision term (Anderson and Witting, 1974),

$$
p^{\mu} \partial_{\mu} f=-(u \cdot p) \frac{\delta f}{\tau_{R}} \Rightarrow f=f_{0}-\frac{\tau_{R}}{(u \cdot p)} p^{\mu} \partial_{\mu} f,
$$

where $\tau_{R}$ is the relaxation time. Expanding the distribution function $f$ about its equilibrium value in powers of space-time gradients, i.e., $f=f_{0}+\delta f^{(1)}+\delta f^{(2)}+\cdots$ and solving Eq. (5) iteratively, we obtain (Jaiswal, 2013a,b),

$$
\delta f^{(1)}=-\frac{\tau_{R}}{u \cdot p} p^{\mu} \partial_{\mu} f_{0}, \quad \delta f^{(2)}=\frac{\tau_{R}}{u \cdot p} p^{\mu} p^{\nu} \partial_{\mu}\left(\frac{\tau_{R}}{u \cdot p} \partial_{\nu} f_{0}\right)
$$

Substituting $\delta f=\delta f^{(1)}$ in the expression for $\pi^{\mu \nu}$ in Eq. (3), performing the integrations, and retaining only first-order terms, we obtain $\pi^{\mu \nu}=2 \tau_{R} \beta_{\pi} \sigma^{\mu \nu}$, where $\beta_{\pi}=4 P / 5$ (Jaiswal, 2013a).

To obtain the second-order evolution equation for shear stress tensor, we rewrite Eq. (5) in the form $\delta \dot{f}=-\dot{f}_{0}-p^{\gamma} \nabla_{\gamma} f /(u \cdot p)-\delta f / \tau_{R}$. Using this expression for $\delta \dot{f}$ in Eq. (4), we obtain

$$
\dot{\pi}^{\langle\mu \nu\rangle}+\frac{\pi^{\mu \nu}}{\tau_{R}}=-\Delta_{\alpha \beta}^{\mu \nu} \int d p p^{\alpha} p^{\beta}\left(\dot{f}_{0}+\frac{1}{u \cdot p} p^{\gamma} \nabla_{\gamma} f\right)
$$

Using Eq. (6) for $\delta f^{(1)}$ and Eq. (3) for derivatives of $\beta$, and keeping terms up to quadratic order in gradients, the second-order shear evolution equation is obtained as (Jaiswal, 2013a)

$$
\dot{\pi}^{\langle\mu \nu\rangle}+\frac{\pi^{\mu \nu}}{\tau_{\pi}}=2 \beta_{\pi} \sigma^{\mu \nu}+2 \pi_{\gamma}^{\langle\mu} \omega^{\nu\rangle \gamma}-\frac{10}{7} \pi_{\gamma}^{\langle\mu} \sigma^{\nu\rangle \gamma}-\frac{4}{3} \pi^{\mu \nu} \theta,
$$

where $\omega^{\mu \nu} \equiv\left(\nabla^{\mu} u^{\nu}-\nabla^{\nu} u^{\mu}\right) / 2$. Using Eq. (3) and (8) in Eq. (6), we arrive at (Bhalerao et al., 2014; Chattopadhyay et al., 2014),

$$
\begin{aligned}
\delta f= & \frac{f_{0} \beta}{2 \beta_{\pi}(u \cdot p)} p^{\alpha} p^{\beta} \pi_{\alpha \beta}-\frac{f_{0} \beta}{\beta_{\pi}}\left[\frac{\tau_{\pi}}{u \cdot p} p^{\alpha} p^{\beta} \pi_{\alpha}^{\gamma} \omega_{\beta \gamma}-\frac{5}{14 \beta_{\pi}(u \cdot p)} p^{\alpha} p^{\beta} \pi_{\alpha}^{\gamma} \pi_{\beta \gamma}\right. \\
& +\frac{(u \cdot p)}{70 \beta_{\pi}} \pi^{\alpha \beta} \pi_{\alpha \beta}-\frac{6 \tau_{\pi}}{5} p^{\alpha} \dot{u}^{\beta} \pi_{\alpha \beta}+\frac{\tau_{\pi}}{3(u \cdot p)} p^{\alpha} p^{\beta} \pi_{\alpha \beta} \theta+\frac{\tau_{\pi}}{5} p^{\alpha}\left(\nabla^{\beta} \pi_{\alpha \beta}\right) \\
& -\frac{3 \tau_{\pi}}{(u \cdot p)^{2}} p^{\alpha} p^{\beta} p^{\gamma} \pi_{\alpha \beta} \dot{u}_{\gamma}+\frac{\tau_{\pi}}{2(u \cdot p)^{2}} p^{\alpha} p^{\beta} p^{\gamma}\left(\nabla_{\gamma} \pi_{\alpha \beta}\right) \\
& \left.-\frac{\beta+(u \cdot p)^{-1}}{4(u \cdot p)^{2} \beta_{\pi}}\left(p^{\alpha} p^{\beta} \pi_{\alpha \beta}\right)^{2}\right]+\mathcal{O}\left(\delta^{3}\right),
\end{aligned}
$$

where the first term on the right-hand side of the above equation corresponds to the first-order correction, $\delta f_{1}$, whereas the terms within square brackets are of second order, $\delta f_{2}$. It is straightforward to show that the form of $\delta f$ in Eq. (9) is consistent with the definition of the shear stress tensor, Eq. (4), and satisfies 
the matching condition $\epsilon=\epsilon_{0}$ and the Landau frame definition $u_{\nu} T^{\mu \nu}=\epsilon u^{\mu}$ at each order (Bhalerao et al., 2014). It is important to note that the experimentally observed $1 / \sqrt{m_{T}}$ scaling of the longitudinal HBT radii (Adcox et al., 2002; Bearden et al., 2001), also predicted by ideal hydrodynamics, is violated by incorporating viscous correction through Grad's 14-moment approximation (Teaney, 2003). However the form of $\delta f$ given in Eq. (9) does not lead to such unphysical effects (Bhalerao et al., 2014). To obtain a thirdorder evolution equation for shear stress tensor, we substitute $\delta f$ from Eq. (9) in Eq. (7). Keeping terms up to cubic order in derivatives, after a straightforward but tedious algebra, we finally obtain a third-order evolution equation for shear stress tensor (Jaiswal, 2013b):

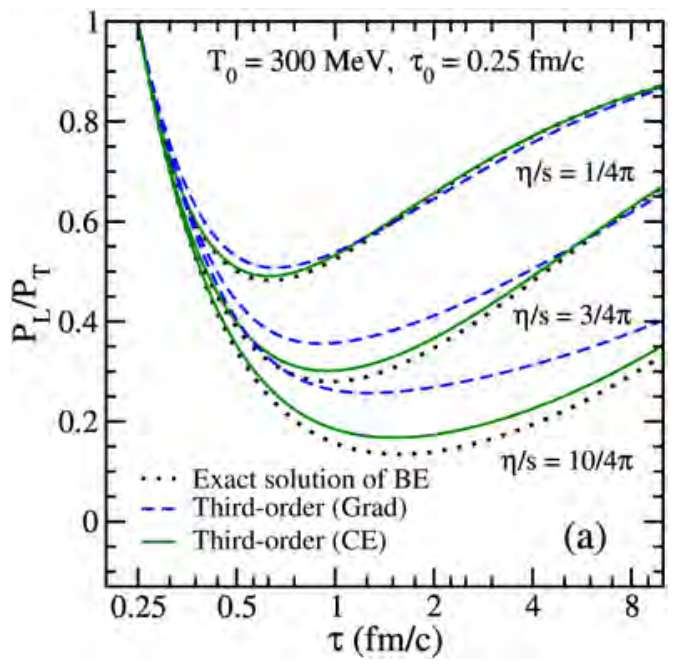

A

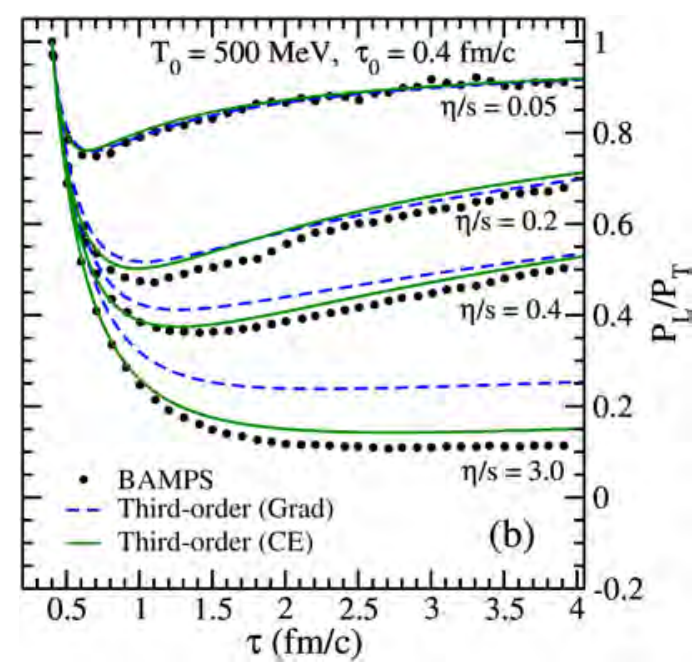

B

Fig. 1: (A): Time evolution of $P_{L} / P_{T}$ obtained using exact solution of Boltzmann equation (dotted line), second-order equations (dashed lines), and third-order equations (solid lines). (B): Time evolution of $P_{L} / P_{T}$ in BAMPS (dots), third-order calculation from entropy method, Eq. (11) (dashed lines), and the present work (solid lines). Both figures are for isotropic initial pressure configuration $\left(\pi_{0}=0\right)$ and various $\eta / s$

$$
\begin{aligned}
\dot{\pi}^{\langle\mu \nu\rangle}= & -\frac{\pi^{\mu \nu}}{\tau_{\pi}}+2 \beta_{\pi} \sigma^{\mu \nu}+2 \pi_{\gamma}^{\langle\mu} \omega^{\nu\rangle \gamma}-\frac{10}{7} \pi_{\gamma}^{\langle\mu} \sigma^{\nu\rangle \gamma}-\frac{4}{3} \pi^{\mu \nu} \theta+\frac{25}{7 \beta_{\pi}} \pi^{\rho\langle\mu} \omega^{\nu\rangle \gamma} \pi_{\rho \gamma} \\
& -\frac{1}{3 \beta_{\pi}} \pi_{\gamma}^{\langle\mu} \pi^{\nu\rangle \gamma} \theta-\frac{38}{245 \beta_{\pi}} \pi^{\mu \nu} \pi^{\rho \gamma} \sigma_{\rho \gamma}-\frac{22}{49 \beta_{\pi}} \pi^{\rho\langle\mu} \pi^{\nu\rangle \gamma} \sigma_{\rho \gamma} \\
& -\frac{24}{35} \nabla^{\langle\mu}\left(\pi^{\nu\rangle \gamma} \dot{u}_{\gamma} \tau_{\pi}\right)+\frac{4}{35} \nabla^{\langle\mu}\left(\tau_{\pi} \nabla_{\gamma} \pi^{\nu\rangle \gamma}\right)-\frac{2}{7} \nabla_{\gamma}\left(\tau_{\pi} \nabla^{\langle\mu} \pi^{\nu\rangle \gamma}\right) \\
& +\frac{12}{7} \nabla_{\gamma}\left(\tau_{\pi} \dot{u}^{\langle\mu} \pi^{\nu\rangle \gamma}\right)-\frac{1}{7} \nabla_{\gamma}\left(\tau_{\pi} \nabla^{\gamma} \pi^{\langle\mu \nu\rangle}\right)+\frac{6}{7} \nabla_{\gamma}\left(\tau_{\pi} \dot{u}^{\gamma} \pi^{\langle\mu \nu\rangle}\right) \\
& -\frac{2}{7} \tau_{\pi} \omega^{\rho\langle\mu} \omega^{\nu\rangle \gamma} \pi_{\rho \gamma}-\frac{2}{7} \tau_{\pi} \pi^{\rho\langle\mu} \omega^{\nu\rangle \gamma} \omega_{\rho \gamma}-\frac{10}{63} \tau_{\pi} \pi^{\mu \nu} \theta^{2}+\frac{26}{21} \tau_{\pi} \pi_{\gamma}^{\langle\mu} \omega^{\nu\rangle \gamma} \theta
\end{aligned}
$$


This is the main result of the present work. We compare the above equation with that obtained in Ref. (El et al., 2010) by invoking the second law of thermodynamics,

$$
\dot{\pi}^{\langle\mu \nu\rangle}=-\frac{\pi^{\mu \nu}}{\tau_{\pi}^{\prime}}+2 \beta_{\pi}^{\prime} \sigma^{\mu \nu}-\frac{4}{3} \pi^{\mu \nu} \theta+\frac{5}{36 \beta_{\pi}^{\prime}} \pi^{\mu \nu} \pi^{\rho \gamma} \sigma_{\rho \gamma}-\frac{16}{9 \beta_{\pi}^{\prime}} \pi_{\gamma}^{\langle\mu} \pi^{\nu\rangle \gamma} \theta,
$$

where $\beta_{\pi}^{\prime}=2 P / 3$ and $\tau_{\pi}^{\prime}=\eta / \beta_{\pi}^{\prime}$. We notice that the right-hand-side of the above equation contains one second-order and two third-order terms compared to three second-order and fourteen third-order terms obtained in Eq. (10). This confirms the fact that the evolution equation obtained by invoking the second law of thermodynamics is incomplete.

\section{Numerical Results and Discussion}

In the following, we consider boost-invariant Bjorken expansion of a massless Boltzmann gas (Bjorken, 1983). We have solved the evolution equations with initial temperature $T_{0}=300 \mathrm{MeV}$ at initial time $\tau_{0}=0.25 \mathrm{fm} / \mathrm{c}$ and with $T_{0}=500 \mathrm{MeV}$ at $\tau_{0}=0.4 \mathrm{fm} / \mathrm{c}$, corresponding to initial conditions of RHIC and LHC, respectively.

Figs. 1(A) and (B) shows the proper time dependence of pressure anisotropy $P_{L} / P_{T} \equiv(P-\pi) /(P+$ $\pi / 2$ ) where $\pi \equiv-\tau^{2} \pi^{\eta \eta}$. In Fig. 1 (A), we observe an improved agreement of third-order results from Chapman-Enskog method (green solid lines) with the exact solution of Boltzmann equation (black dotted lines) (Florkowski et al., 2013) as compared to third-order results from Grad's method (blue dashed lines). In Fig. 1(B) we notice that while the results from Grad's method (blue dashed lines) overestimate the pressure anisotropy for $\eta / s>0.2$, those obtained in the present work (green solid lines) are in better agreement with the results of the parton cascade BAMPS (black dots) (El et al., 2010).

\section{Summary}

To summarize, we have derived a third-order evolution equation for the shear stress tensor from kinetic theory. We iteratively solved the Boltzmann equation in relaxation time approximation to obtain the nonequilibrium distribution function up to second-order in gradients. Using this form of the non-equilibrium distribution function, instead of Grad's 14-moment approximation, the evolution equation for shear tensor was derived directly from its definition. Within one-dimensional scaling expansion we demonstrated that the third-order viscous hydrodynamic equation derived here provides a very good approximation to the exact solution of Boltzmann equation. We also showed that our results are in better agreement with BAMPS compared to third-order viscous hydrodynamics derived using Grad's approximation. 


\section{References}

1. Adcox K et al, [PHENIX Collaboration] (2002) Transverse mass dependence of two pion correlations in $\mathrm{Au}+\mathrm{Au}$ collisions at $\mathrm{S}(\mathrm{NN}) * *(1 / 2)=130-\mathrm{GeV}$, Phys Rev Lett 88192302 [nucl-ex/0201008]

2. Anderson JL and Witting HR (1974) A relativistic relaxation-time for the Boltzmann equation, Physica 74466

3. Bearden IG et al., [NA44 Collaboration] (2001) Two kaon correlations in central $\mathrm{Pb}+\mathrm{Pb}$ collisions at $158 \mathrm{~A}$ GeV / c, Phys Rev Lett 87112301

4. Bhalerao RS, Jaiswal A, Pal S and Sreekanth V (2013) Particle production in relativistic heavy-ion collisions: A consistent hydrodynamic approach, Phys Rev C 88044911

5. Bhalerao RS, Jaiswal A, Pal S and Sreekanth V (2014) Relativistic viscous hydrodynamics for heavy-ion collisions: A comparison between the Chapman-Enskog and Grad methods, Phys Rev C 89054903

6. Bjorken JD (1983) Highly Relativistic Nucleus-Nucleus Collisions: The Central Rapidity Region, Phys Rev D 27140

7. Chattopadhyay C, Jaiswal A, Pal S and Ryblewski R (2014) Relativistic third-order viscous corrections to the entropy four-current from kinetic theory, arXiv:1411.2363 [nucl-th]

8. Danielewicz P and Gyulassy M (1985) Dissipative Phenomena in Quark Gluon Plasmas, Phys Rev D 3153

9. de Groot SR, van Leeuwen WA and van Weert Ch G (1980) Relativistic Kinetic Theory - Principles and Applications, North-Holland, Amsterdam

10. Denicol GS, Koide T and Rischke DH (2010) Dissipative relativistic fluid dynamics: a new way to derive the equations of motion from kinetic theory, Phys Rev Lett 105162501

11. Denicol GS, Niemi H, Molnar E and Rischke DH (2012) Derivation of transient relativistic fluid dynamics from the Boltzmann equation, Phys Rev D 85114047

12. Eckart C (1940) The Thermodynamics of Irreversible Processes. 1. The Simple Fluid, Phys Rev 58267

13. El A, Muronga A, Xu Z and Greiner C (2009) Shear viscosity and out of equilibrium dissipative hydrodynamics, Phys Rev C 79044914

14. El A, Xu Z and Greiner C (2010) Third-order relativistic dissipative hydrodynamics, Phys Rev C 81041901

15. Florkowski W, Ryblewski R and Strickland M (2013) Anisotropic Hydrodynamics for Rapidly Expanding Systems, Nucl Phys A 916249

16. Huovinen P and Molnar D (2009) The Applicability of causal dissipative hydrodynamics to relativistic heavy ion collisions, Phys Rev C 79014906

17. Israel W and Stewart JM (1979) Transient relativistic thermodynamics and kinetic theory, Ann Phys 118341

18. Jaiswal A, Bhalerao RS and Pal S (2013a) New relativistic dissipative fluid dynamics from kinetic theory, Phys Lett B $\mathbf{7 2 0} 347$

19. Jaiswal A, Bhalerao RS and Pal S (2013b) Boltzmann equation with a nonlocal collision term and the resultant dissipative fluid dynamics, J Phys Conf Ser 422012003

20. Jaiswal A, Bhalerao RS and Pal S (2013c) Complete relativistic second-order dissipative hydrodynamics from the entropy principle, Phys Rev C 87021901 
21. Jaiswal A (2013a) Relativistic dissipative hydrodynamics from kinetic theory with relaxation time approximation, Phys Rev C 87051901

22. Jaiswal A (2013b) Relativistic third-order dissipative fluid dynamics from kinetic theory, Phys Rev C 88021903

23. Jaiswal A (2014) Relaxation-time approximation and relativistic third-order viscous hydrodynamics from kinetic theory, Nucl Phys A [arXiv:1407.0837 [nucl-th]]

24. Jaiswal A, Ryblewski R and Strickland M (2014) Transport coefficients for bulk viscous evolution in the relaxation time approximation, Phys Rev C 90044908

25. Landau LD and Lifshitz EM (1987) Fluid Mechanics, Butterworth-Heinemann, Oxford

26. Martinez M and Strickland M (2009) Constraining relativistic viscous hydrodynamical evolution, Phys Rev C 79044903

27. Muronga A (2004) Causal theories of dissipative relativistic fluid dynamics for nuclear collisions, Phys Rev C 69034903

28. Teaney D (2003) The Effects of viscosity on spectra, elliptic flow, and HBT radii, Phys Rev C 68034913. 